\title{
Determining the balance between nutrition and sustainability of 173 food products using stepwise optimisation
}

\author{
Roline Broekema and Hans Blonk \\ Blonk Consultants, Gouda, Netherlands
}

\begin{abstract}
Introduction

The balance between nutrition and environmental impact is key for determining whether a product is future-proof. This balance refers to the Sustainability Nutrition Balance (SNB). A product that provides nutrients which improve the quality of the current diet with a low sustainability impact has a better SNB-score than a product that contains nutrients that we tend to consume in excess (like salt or saturated fat) and/ or with a high sustainability impact. For 173 food products, we calculated the SNB-score. These scores are valuable to guide product development in the direction of future healthy and sustainable diets.
\end{abstract}

\section{Materials and methods}

Based on the European Food Safety Authority (EFSA) Food Consumption Database 173 products were selected, making up a European average diet. EFSA Food Composition Database was used to gather the nutritional properties of the products, like energy, dietary fibre, vitamin D, magnesium. About 60 nutritional properties were considered. The environmental impact of the products was calculated using Life Cycle Assessment (LCA) method. EFSA's Dietary Reference Values for Nutrients and Tolerable Upper Intake Values for Vitamins and Minerals were consulted to determine the nutritional constraints for a healthy diet.

To analyse the SNB-score the amount of a product in the diet is varied in steps. At every step the diet is optimised for nutritional constraints, using quadratic programming. This allows identification of trends in terms of the environmental impact of the diet on indicators like climate change and land use. An increase of impact with an increase in amount of product leads to a higher SNB-score. This indicates that the group of products which is nutritionally equivalent to the product of focus is a more sustainable alternative. The lower the SNB-score, the more future-proof a product is in terms of sustainability and nutrition.

\section{Results and discussion}

This analysis has led to a palette of SNB-scores for the 173 products in the European average diet. Meat tends to have a higher SNB-score than dairy and whole grain products can have a negative SNB-score. This means the environmental impact of the product decreases when whole grains are consumed in increasing amounts. The palette of SNB-scores serves as a benchmark for product development. To create more future-proof products the SNB-scores can be improved by changing the nutritional or environmental profile by altering recipes or production processes.

\section{Conflict of Interest}

There is no conflict of interest 